\title{
MicroRNA-126 Priming Enhances Functions of Endothelial Progenitor Cells under Physiological and Hypoxic Conditions and Their Therapeutic Efficacy in Cerebral Ischemic Damage
}

\author{
Qunwen Pan, ${ }^{1}$ Jieyi Zheng, ${ }^{1}$ Donghui Du, ${ }^{1}$ Xiaorong Liao, ${ }^{1}$ Chunlian $\mathrm{Ma}^{2}{ }^{2}$ Yi Yang $\mathbb{D},{ }^{2}$ \\ Yanyu Chen, ${ }^{1}$ Wangtao Zhong $\mathbb{D}^{1}{ }^{1}$ and Xiaotang $M a \mathbb{D}^{1}$ \\ ${ }^{1}$ Guangdong Key Laboratory of Age-Related Cardiac and Cerebral Diseases, Institute of Neurology, Affiliated Hospital of Guangdong \\ Medical University, Zhanjiang 524001, China \\ ${ }^{2}$ College of Health Science, Wuhan Sports University, Wuhan 430079, China
}

Correspondence should be addressed to Wangtao Zhong; 158980116@qq.com and Xiaotang Ma; mxtgdmc@163.com

Received 16 December 2017; Accepted 9 January 2018; Published 11 April 2018

Academic Editor: Zhen Yang

Copyright (C) 2018 Qunwen Pan et al. This is an open access article distributed under the Creative Commons Attribution License, which permits unrestricted use, distribution, and reproduction in any medium, provided the original work is properly cited.

\begin{abstract}
Endothelial progenitor cells (EPCs) have shown the potential for treating ischemic stroke (IS), while microRNA-126 (miR-126) is reported to have beneficial effects on endothelial function and angiogenesis. In this study, we investigated the effects of miR-126 overexpression on EPCs and explore the efficacy of miR-126-primed EPCs (EPC ${ }^{\text {miR-126}}$ ) in treating IS. The effects of miR-126 overexpression on EPC proliferation, migratory, tube formation capacity, reactive oxygen species (ROS) production, and nitric oxide (NO) generation were determined. In in vivo study, the effects of EPC ${ }^{\text {mik-126 }}$ on the cerebral blood flow (CBF), neurological deficit score (NDS), infarct volume, cerebral microvascular density (cMVD), and angiogenesis were determined. Moreover, the levels of circulating EPCs (cEPCs) and their contained miR-126 were measured. We found (1) miR-126 overexpression promoted the proliferation, migration, and tube formation abilities of EPCs; decreased ROS; and increased NO production of EPCs via activation of PI3K/Akt/eNOS pathway; (2) EPC ${ }^{\text {miR-126 }}$ was more effective than EPCs in attenuating infarct volume and NDS and enhancing $\mathrm{CMVD}, \mathrm{CBF}$, and angiogenesis; and (3) infusion of EPC ${ }^{\text {miR-126 }}$ increased the number and the level of miR-126 in cEPCs. Our data indicate that miR-126 overexpression enhanced the function of EPCs in vitro and in vivo.
\end{abstract}

\section{Introduction}

Cerebral ischemic stroke is one of the leading causes of death and disability in the world. Endothelial repair and neovascularization are important for functional recovery from ischemic stroke [1-4]. Endothelial progenitor cells (EPCs) could differentiate into mature vascular endothelial cells (ECs), playing important roles in maintaining vascular homeostasis and promoting angiogenesis [3]. Under vascular ischemic injury, EPCs mobilize from the bone marrow (BM) to injury area, participating in the neovascularization [1]. Reduced number and impaired function of circulating EPCs (cEPCs) are reported to be correlated to worse clinical outcome in patients with ischemic stroke [5]. EPC transfusion has shown potential therapeutic effects on ischemic stroke [6]. However, under such pathological environment, the function of transfused EPCs is usually impaired by existing risk factors [6-8]. Therefore, exploring approaches to combine autologous cell therapy with ex vivo genetic manipulations to maintain/ improve their function is critical for ischemic stroke therapy.

MicroRNAs (miRs), highly conserved, single-stranded 18-25 nucleotide noncoding RNAs, could regulate target gene expression by degrading the messenger RNAs (mRNAs) and inhibit the translation of protein [9]. miR-126, highly expressed in ECs and EPCs, is a multifunctional miR playing important roles in regulating vascular integrity and promoting angiogenesis. Several studies have demonstrated that miR-126 could enhance the proliferation and migration abilities of ECs $[10,11]$. However, circulating miR-126 has been shown to be reduced in vascular diseases including stroke 
and coronary artery disease $[12,13]$. Moreover, miR-126 was downregulated in EPCs under hypoxia condition [14]. Previous study has demonstrated that knockdown of miR-126 expression impairs ischemia-induced vessel reparation [15]. Thus, we hypothesize that overexpression of miR-126 could rescue/promote functions of EPCs under hypoxic condition and enhance the therapeutic efficacy of EPCs in ischemic stroke. Meanwhile, miR-126 has been reported to regulate angiogenic process and EC/EPC function via phosphorylated phosphatidylinositol-3-kinase (PI3K)/Akt/endothelial nitric oxide synthase (eNOS) pathway $[16,17]$. Downregulation of miR-126 in EPCs impairs their function through PI3K/ Akt/eNOS signal pathway [18]. Therefore, we aimed to study the inner mechanism of miR-126 overexpression in the modulation of EPC survival and functions by regulating the PI3K/ Akt/eNOS pathway.

In the present study, we investigated the role of miR-126 overexpression in regulating functions of normoxia- or hypoxia-injured EPCs. The underlying mechanism of PI3K/ Akt/eNOS pathway was explored. Moreover, we determined whether transfusion of miR-126-primed EPCs (EPC ${ }^{\text {miR-126 }}$ ) is more effective in treating ischemic stroke in mouse model. The level of cEPCs and their contained miR-126 was also examined in EPC-transfused MCAO mice.

\section{Materials and Methods}

2.1. Culture and Characterization of EPCs. EPCs were generated from C57BL6 mice and characterized as previously reported [19]. After 7 days of culture, the cells that double positive for Di-acLDL and Bs-Lectin staining were considered as early outgrowth EPCs.

2.2. EPC Transfection. The lentivirus-carrying green fluorescent protein (GFP) marker for gene expression of murine miR-126 (Lv-miR-126) or scrambled control (Lv-NC) were purchased from Genepharma (Shanghai, China). To obtain overexpressed miR-126 (EPC $\left.{ }^{\text {miR-126}}\right)$ and control EPCs $\left(\mathrm{EPC}^{\mathrm{NC}}\right)$, the EPCs were transfected with Lv-miR-126 or Lv-NC as previously described [20]. In brief, the EPCs were incubated with EPC culture medium containing the lentivirus for $24 \mathrm{~h}$. Transduction efficiency (the expression of miR-126 in EPCs) was quantified by RT-PCR.

2.3. Hypoxia Model of EPCs. Hypoxia injury model of EPCs was conducted as previously described [21, 22]. In brief, EPC $^{\text {miR-126 }}$ or EPC EC $^{\mathrm{NC}}$ were subjected to hypoxia $\left(\mathrm{O}_{2} / \mathrm{N}_{2} /\right.$ $\left.\mathrm{CO}_{2}, 1 / 94 / 5\right)$ for $72 \mathrm{~h}$. The cells cultured under normoxic conditions for $72 \mathrm{~h}$ were set as the control. Afterwards, the EPCs were used for functional analyses (proliferation, migration, and tube formation).

2.4. Real-Time Polymerase Chain Reaction (RT-PCR). The level of miR-126 in the EPCs was determined using Hairpin-it $^{\mathrm{TM}}$ miRs RT-PCR Quantitation kit (GenePharma, Shanghai, China) based on manufactory instruments. PCR primer are as follows: 5-TATGGTTGTTCTCGACTCCTT CAC-3 and 5-TCGTCTGTCGTACCGTGAGTAAT-3 for miR126 and 5-CTCGCT TCGGCAGCACA-3 and 5-AACG CT TCACGAATTTGCGT-3 for U6. Quantitative real-time
PCR was conducted on a LightCycler 96 System (Roche Diagnostics, Switzerland). U6 was used for normalizing the data of miR-126 expression. Relative expression of miR-126 was calculated by using the $2^{-\Delta \Delta C T}$ method.

2.5. EPC Proliferation Analysis. The proliferation ability of EPCs was measured by MTT (3-(4,5-dimethylthiazyol-2yl)2,5-diphenyltetrazolium bromide) $(5 \mathrm{mg} / \mathrm{ml}$, Sigma) assay. The EPCs were seeded at $2 \times 10^{3} /$ well in 96 -well plate with $100 \mu \mathrm{L}$ EGM-2 culture medium. MTT solution $(20 \mu \mathrm{L})$ was added and incubated with cells for $4 \mathrm{~h}$ at $37^{\circ} \mathrm{C}$, then $150 \mu \mathrm{L}$ DMSO was added to each well and incubated with the cells for $20 \mathrm{~min}$ at $37^{\circ} \mathrm{C}$. The optical density (OD) value of cells was read at $490 \mathrm{~nm}$ using a microplate reader (BioTek). Cells in triplicate wells were examined at each time point, and the experiment was repeated three times.

2.6. EPC Migration Analysis. The migration of EPCs was measured as previously described [23]. Briefly, a scratch was made through the cultured EPCs treated with hypoxia or normoxia (control). After $16 \mathrm{~h}$ cultivation, the invasion of cells into the scratched area was observed by an inverted microscope. Quantitative analysis of migration was calculated as the equation: (cell-free area at $0 \mathrm{~h}$ - cell-free area at $16 \mathrm{~h}) /$ cell free area at $0 \mathrm{~h} \times 100 \%$.

2.7. EPC Tube Formation Analysis. The tube formation ability was measured by using the tube formation assay kit (Chemicon) as we previously described [23]. Briefly, the EPCs were placed $\left(1 \times 10^{4}\right.$ cells/well $)$ onto the surface of the EC matrix and incubated with EPC culture medium for $12 \mathrm{~h}$ at $37^{\circ} \mathrm{C}$. Four representative fields were taken, and the average of complete tubes formed by EPCs in the fields was counted.

2.8. ROS Analysis. Dihydroethidium (DHE) (Beyotime, China) staining was used to measure the intracellular ROS production as we previously described [24]. The EPCs treated with hypoxia or normoxia (control) were incubated with DHE solution $(5 \mu \mathrm{M})$ at $37^{\circ} \mathrm{C}$ for $2 \mathrm{~h}$. Flow cytometry was used to analyze the level of ROS in EPCs. For signal pathway study, LY294002 (PI3K inhibitor; $20 \mu \mathrm{M}$ ) was prior added to culture medium for $2 \mathrm{~h}$.

2.9. NO Analysis. Total NO production of EPCs treated with hypoxia or normoxia was detected by using a Nitric Oxide Assay kit (Beyotime) as we previously described [24]. For pathway blocking experiments, the cells were preincubated with LY294002 $(20 \mu \mathrm{M})$ for $2 \mathrm{~h}$.

2.10. Western Blot Analysis. The protein of EPCs were extracted with cell lysis buffer (Applygen Technologies Company, Beijing) supplemented with protease inhibitor tablet (Thermo Scientific). Protein lysates were electrophoresed through SDS-PAGE gels and transferred onto PVDF membranes. The membranes were blocked with $5 \%$ nonfat milk for $1 \mathrm{~h}$ and incubated with primary antibodies against beta actin $(1: 1000$, EarthOx, San Francisco, CA, USA), PI3 kinase p110a (1:1000, CST, USA), Akt (1:5000, CST, USA), p-Akt (1:500, CST, USA), and p-eNOS (1:1000, 
Invitrogen, USA). Blots were developed with ECL solution (Amersham, Sweden).

\section{Animals and Procedure}

3.1. Animals. Adult C57BL6/J mice (6-8 weeks of age; weight ranges from 20-24g) were purchased from the Animal Experiment Center of Guangdong Province (Guangzhou, China) and housed in the Animal Care Facility at the Guangdong Medical University. The mice were maintained in a pathogen-free environment with free access to food and water on a $12 \mathrm{~h}$ light/dark cycle before and after surgery. All surgery was performed under $2.5 \%$ isoflurane anesthesia, and all efforts were made to minimize pain and distress. All experimental procedures were approved by the Laboratory Animal Care and Use Committees at Guangdong Medical University.

3.2. Middle Cerebral Artery Occlusion Surgery (MCAO) and EPC Transfusion. The mice were included and random divided into sham, MCAO, $\mathrm{EPC}^{\mathrm{NC}}$, and EPC ${ }^{\mathrm{miR}-126}$ groups. Focal ischemic stroke and sham-operated mice were carried out as we reported previously [25]. Two hours after MCAO, the mice ( $n=10$ /group) were administrated via the tail vein with phosphate-buffered saline (PBS, vehicle), $\mathrm{EPC}^{\mathrm{NC}}$, or $\mathrm{EPC}^{\mathrm{miR}-126}\left(2 \times 10^{6}\right.$ cells $/ 100 \mu \mathrm{L}$ in PBS $)$. The EPCs were donated from C57BL6 mice. Three days after EPC transfusion, the brain samples of mice were used for the measurements of NDS, cerebral blood flow (CBF), infarct volume, and cerebral microvascular density (cMVD) in the periinfarct area. The blood samples were used for the analyses of the numbers and the level of miR-126 of cEPCs. In addition, seven days after EPC transfusion, the angiogenesis in the peri-infarct area was measured.

3.3. Measurements of Neurological Deficit Score. On day 3, the NDS was evaluated as we previously described by using the 5point scale method [25].

3.4. Measurement of $C B F$. The $\mathrm{CBF}$ of mice from various groups was measured by the PeriCam PSI System (Perimed, Sweden) as we previously described [25].

3.5. Measurements of Infarct Volume. On day 3 after EPC transfusion, the infarct volume of each mouse was measured by using $2 \%$ 2,3,5-triphenyltetrazolium chloride (TTC) staining as we previously described [25].

3.6. Immunofluorescence Analysis. cMVD in the peri-infarct area was measured by using immunofluorescence staining as described in a previous study [26]. For angiogenesis, BrdU (IP, $65 \mu \mathrm{g} / \mathrm{g}$ per day) was administered by intraperitoneal injection after EPC infusion for 7 continuous days [19] and determined by staining with BrdU and CD31. Specifically, the brain coronal sections were incubated with $\operatorname{BrdU}(1: 50$; Abcam) and/or CD31 (1:50; BD Biosciences) antibody overnight at $4^{\circ} \mathrm{C}$. On the second day, the brain sections were reacted with FITC-BrdU (green) and Cy3-CD31 (red) secondary antibodies (1:250; Invitrogen) for 30 minutes at room temperature in the dark. The positive cells in the peri-infarct area of each section were observed using a confocal microscope (Leica, TCS SP5II, Germany). Angiogenesis was determined as BrdU+CD31+ cells according to previous report [19].

3.7. Isolation and Analysis of cEPCs. The level of cEPCs was determined by flow cytometry as a previous study [27]. Briefly, circulating MNCs were isolated from the peripheral blood and stained with antimouse CD34-PE (AbD Serotec, Raleigh, NC) and VEGFR2-PE-Cy3 (BD, Bioscience) antibodies for $30 \mathrm{~min}$ at RT. Isotype-matched (IgG) nonspecific antibodies served as negative controls. After incubation, labeled EPCs were washed with PBS and resuspended with $100 \mu \mathrm{L}$ of PBS for flow cytometric analysis. The EPCs were defined as CD34+VEGFR+ cells. The number of cEPCs was described as the number of cells per microliters of whole blood.

cEPCs were further isolated by magnetic cell sorting (MACS) (Miltenyi Biotec $\mathrm{GmbH}$ ) according to the manufacturer's instruction. The collected MNCs were incubated with $10 \mu \mathrm{L}$ of biotin-conjugated anti-CD34 antibody (Miltenyi Biotec) in a $100 \mu \mathrm{L}$ reaction volume for $10 \mathrm{~min}$, followed by adding $10 \mu \mathrm{L}$ of antibiotin microbeads (Miltenyi Biotec) for $15 \mathrm{~min}$. Then, the microbead-labeled MNCs were separated by using PureProteome Magnetic Stand (Millipore). After an overnight magnet separation, the microbeadbound MNCs were resuspended with $100 \mu \mathrm{L}$ PBS. The multisort release reagent $(10 \mu \mathrm{L}$; Miltenyi Biotec) was used to cleave off the microbeads. After $10 \mathrm{~min}$, the cells were washed from the released fraction carefully by adding $1 \mathrm{~mL}$ of PBS and centrifuged at $300 \mathrm{~g}$ for $10 \mathrm{~min}$. The collected cells were incubated with $10 \mu \mathrm{L}$ of biotin-conjugated anti-VEGFR2 antibody (Miltenyi Biotec) in a $100 \mu \mathrm{L}$ reaction volume for $10 \mathrm{~min}$, followed by adding $10 \mu \mathrm{L}$ of antibiotin microbeads (Miltenyi Biotec) for $15 \mathrm{~min}$. Then, the labeled cells were proceeded to magnetic separation as mentioned above. The isolated CD34+VEGFR2+ cells were considered as cEPCs and used for miR-126 expression detection.

3.8. Statistical Analysis. All data were expressed as mean \pm SD. Multiple comparisons were analyzed by one- or two-way ANOVA followed by Tukey post hoc tests. SPSS 23.0 statistical software was used for analyzing the data. For all measurements, a $p<0.05$ was considered statistically significant.

\section{Results}

4.1. Characterization of EPCs and miR-126 Expression in EPCs. As shown in Figure 1(a), the EPCs were defined as Di-acLDL and Bs-Lectin double-staining cells. Our qRTPCR results showed that hypoxia significantly decreased miR-126 expression in EPCs (versus control; $p<0.05$; Figure 1(b)). Not surprising, miR-126 transduction significantly increased the level of miR-126 in both vehicle and hypoxia-injured EPCs (versus EPC ${ }^{\mathrm{NC}} ; p<0.05$; Figure 1(b)).

4.2. miR-126 Promoted the Proliferation of EPCs. MTT assay showed that hypoxia markedly decreased the proliferation of EPCs (versus control; $p<0.05$; Figure 1(c)). Overexpression of miR-126 increased the proliferation of 

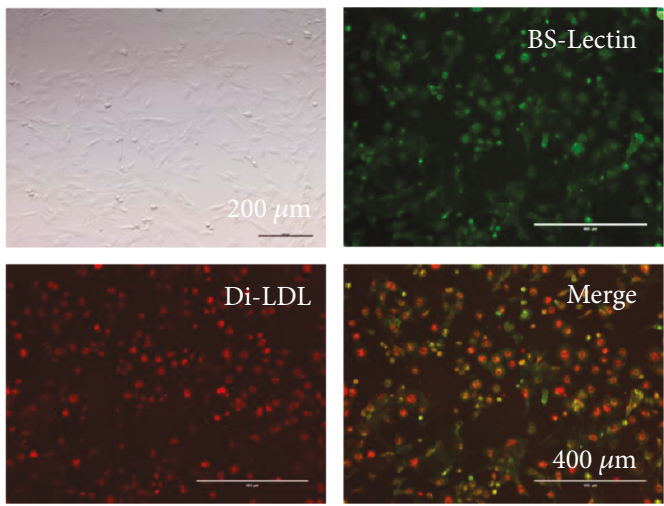

(a)

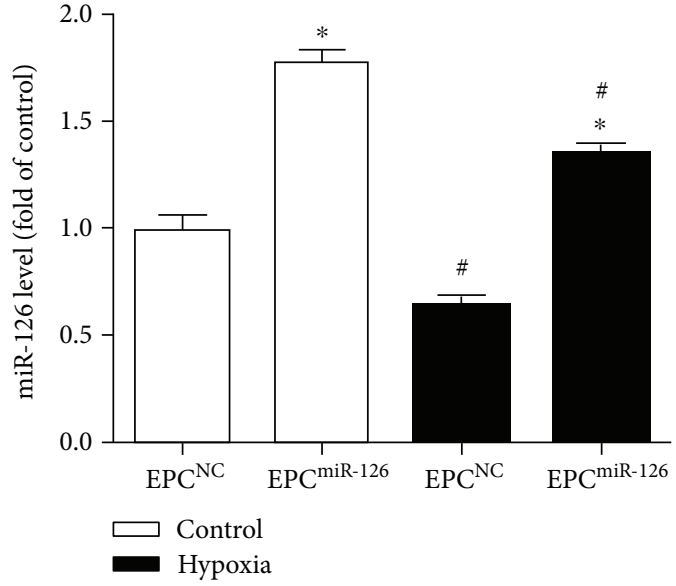

(b)

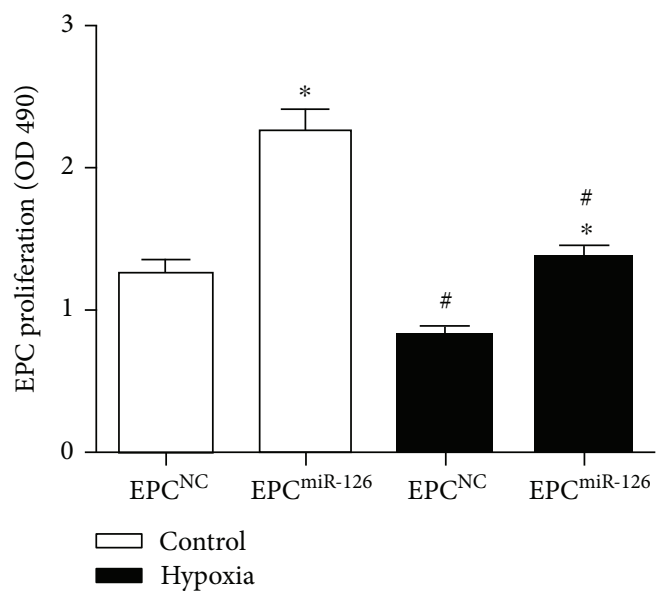

(c)

FIGURE 1: Characterization and transfection efficiency of EPCs and the effect of miR-126 overexpression on the proliferation ability of EPCs and hypoxia-injured EPCs. (a) Representative pictures showing cultured EPCs under transmitter light and fluorescent light by doublestaining analysis. Red: Di-acLDL uptaking; green: Bs-Lectin staining; yellow: Di-acLDL and Bs-Lectin positive cells as EPCs. Scale bar: $400 \mu \mathrm{m}$. (b) qRT-PCR analysis of miR-126 expression in EPCs after lentivirus-miR-126 transfection. (c) MTT assay of EPC proliferation. ${ }^{*} p<0.05$ versus $\mathrm{EPC}^{\mathrm{NC}} ;{ }^{\#} p<0.05$ versus control, $n=3$ /group.

EPCs in both control and hypoxia groups (versus $\mathrm{EPC}^{\mathrm{NC}}$; $p<0.05$; Figure 1(c)).

4.3. miR-126 Promoted the Migration of EPCs. As shown in Figure 2, the migration of EPCs was significantly decreased under hypoxia (versus control; $p<0.05$; Figure 2). Overexpression of miR-126 significantly improved the migration of EPCs in control and hypoxia groups (versus $\mathrm{EPC}^{\mathrm{NC}} ; p<$ 0.05; Figure 2).

4.4. miR-126 Increased the Tube Formation Ability of EPCs. As shown in Figure 3, the tube formation ability of hypoxia-treated EPCs was markedly decreased (versus control; $p<0.05$; Figure 3). After miR-126 overexpression, the tube formation ability of EPCs was significantly increased in both vehicle and hypoxia-treated groups (versus $\mathrm{EPC}^{\mathrm{NC}}$; $p<0.05$; Figure 3).

4.5. miR-126 Decreased ROS Production and Increased NO Production of EPCs via PI3K/Akt/eNOS Pathway. Our results showed that ROS production significantly increased while NO production markedly decreased in hypoxia-treated EPCs (versus control; $p<0.05$; Figure 4(a)), whereas miR-126 transduction increased NO production and decreased ROS production in both control and hypoxia-treated groups

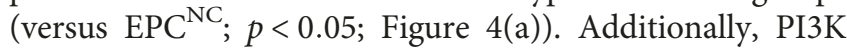
inhibitor inhibited the beneficial effects of miR-126 on increasing NO production and decreasing ROS production of EPCs (versus EPC ${ }^{\text {miR-126}} ; p<0.05$; Figure 4(a)).

Western blot results showed that the levels of PI3K, pAkt/Akt, and p-eNOS in hypoxia-treated EPCs were decreased (versus control; $p<0.05$; Figure 4(b)), whereas miR-126 transduction increased the levels of PI3K, p-Akt/ Akt, and p-eNOS in control or hypoxia-injured EPCs (versus $\mathrm{EPC}^{\mathrm{NC}} ; p<0.05$; Figure $\left.4(\mathrm{~b})\right)$. Moreover, PI3K pathway inhibitor alleviated the effects of miR-126 on increasing p-Akt/Akt and p-eNOS expression (versus EPC ${ }^{\text {miR-126; }}$ $p<0.05$; Figure 4(b)). These data indicated that PI3K/Akt/ eNOS pathway was implicated in the effects of miR-126 on NO production and ROS generation in EPCs.

4.6. miR-126 Enhanced the Therapeutic Effects of EPCs on Decreasing Cerebral Injury and Improving Neurological Deficits and CBF in MCAO Mice. As shown in Figure 5, 

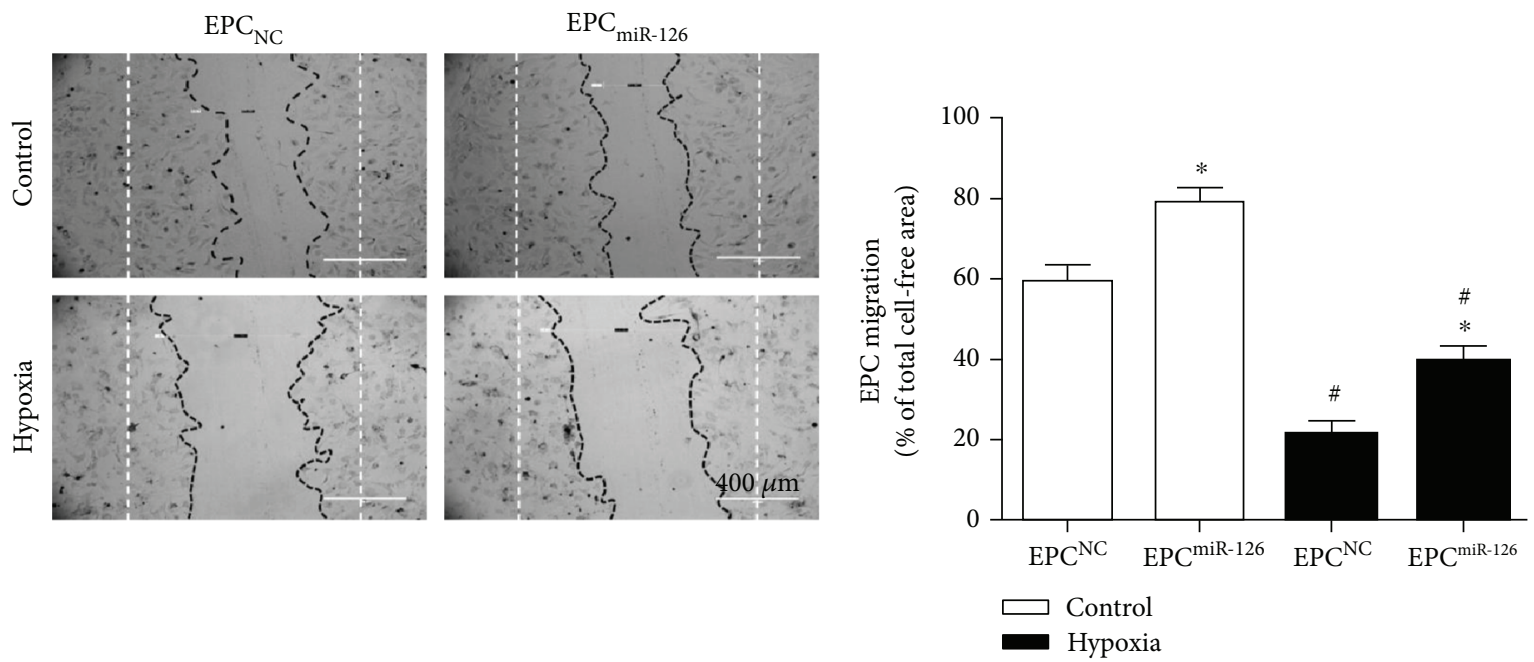

(a)

(b)
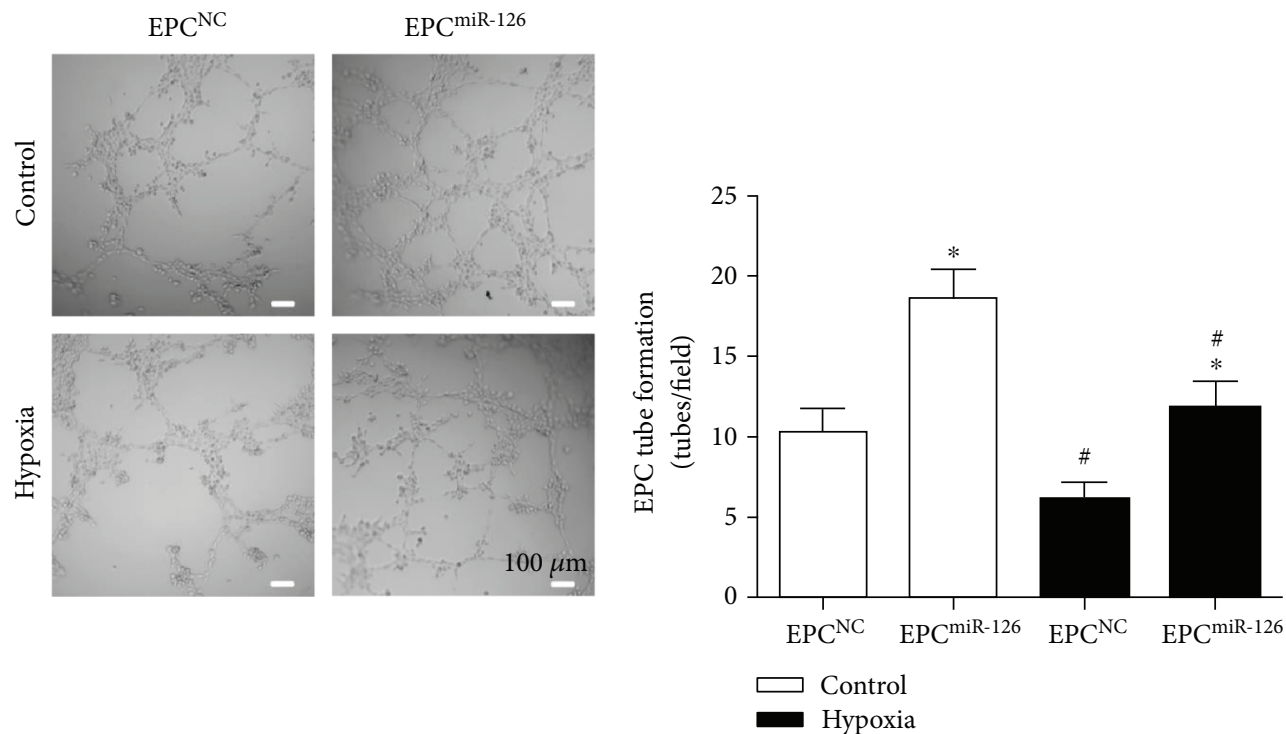

(c)

(d)

FIGURE 2: Effects of miR-126 overexpression on the migration and tube formation of EPCs and hypoxia-injured EPCs. (a) Representative image of EPC migrations. Scale bar: $400 \mu \mathrm{m}$. (b) Summarized data on migration of EPCs. (c) Representative image of EPC tube formation. (d) Summarized data on tube formation. ${ }^{*} p<0.05$ versus $\mathrm{EPC}^{\mathrm{NC}}$; ${ }^{\#} p<0.05$ versus control, $n=3$ /group.

the EPCs significantly reduced infarct volume and NDS (versus vehicle, $p<0.05$; Figures 5(a) $-5(\mathrm{c})$ ). Meanwhile, the EPCs also improved the CBF (versus vehicle; $p<0.01$; Figures 5(d), 5(e)). What's more, miR-126 overexpression significantly promoted the therapeutic effects of EPCs on increasing CBF, reducing infarct volume and NDS of MCAO mice (versus $\mathrm{EPC}^{\mathrm{NC}} ; p<0.05$ ).

4.7. Infusion of miR-126-Primed EPCs Enhanced the Efficacy in Increasing $c M V D$ and Promoted Angiogenesis in the Peri-Infarct Area of Ischemic Damage. EPCs increased the cMVD in the peri-infarct area in MCAO mice (versus vehicle; $p<0.05$; Figure 6). As expected, the transfusion of miR-126-primed EPCs enhanced the efficacy (versus EPC ${ }^{\mathrm{NC}}$; $p<0.05$; Figure 6). Moreover, as shown in Figure 6, EPC transfusion increased angiogenesis (versus vehicle; $p<0.05$; Figure 6). The transfusion of miR-126-primed EPCs showed better efficacy in promoting angiogenesis (versus $\mathrm{EPC}^{\mathrm{NC}}$; $p<0.05$; Figure 6).

4.8. miR-126-Primed EPCs Increased the Level of cEPCs and Their Contained miR-126. As shown in Figure 7, the infusion of EPCs increased the levels of cEPCs after 3-day transfusion (versus vehicle; $p<0.05$; Figure $7(\mathrm{a})$ ). Infusion of miR-126primed EPCs further increased the level of cEPCs at this time point (versus vehicle; $p<0.05$; Figure $7(\mathrm{a})$ ). Furthermore, the infusion of EPCs increased miR-126 expression in cEPCs (versus vehicle; $p<0.05$; Figure $7(\mathrm{~b})$ ). In addition, the 


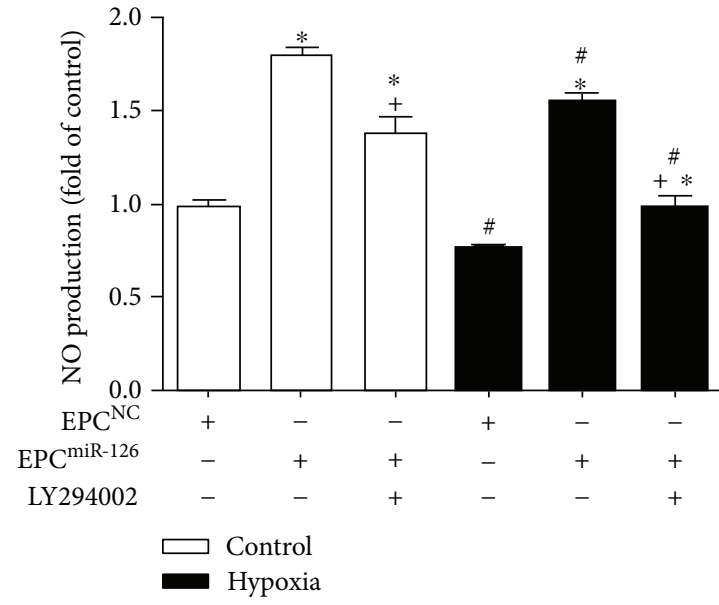

(a)

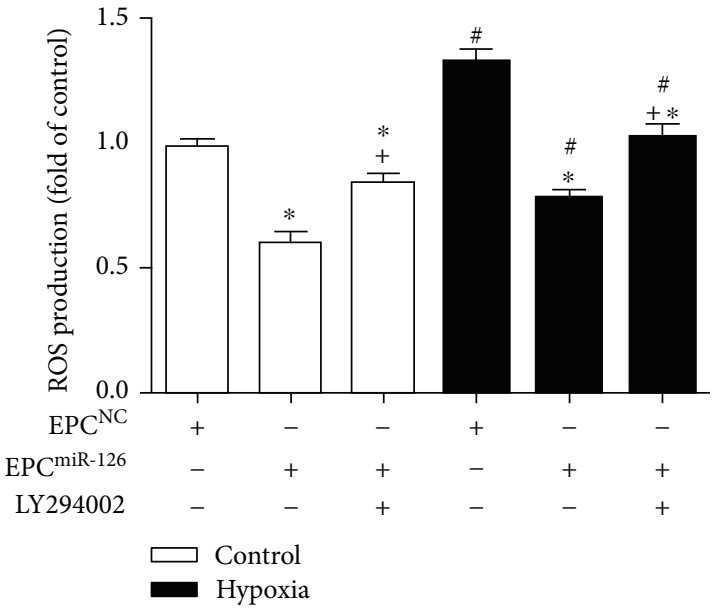

(b)

FIGURE 3: Effects of miR-126 overexpression on ROS and NO production in EPCs and hypoxia-injured EPCs. (a) NO generation of EPCs. (b) ROS production of EPCs. ${ }^{*} p<0.05$ versus $\mathrm{EPC}^{\mathrm{NC}} ;{ }^{+} p<0.05$ versus $\mathrm{EPC}{ }^{\mathrm{miR}-126} ;{ }^{\#} p<0.05$ versus control, $n=3$ /group.

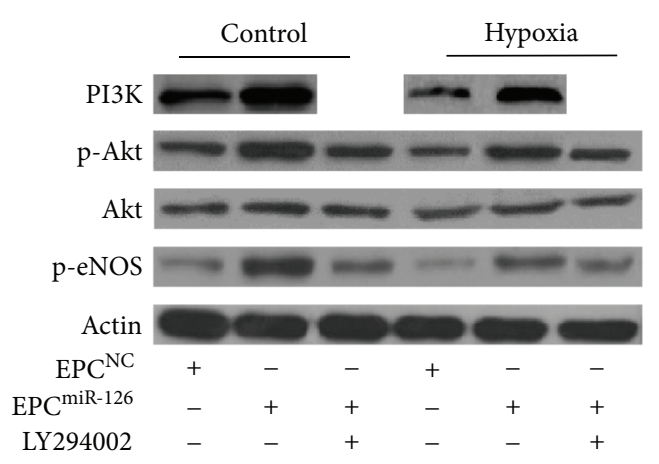

(a)

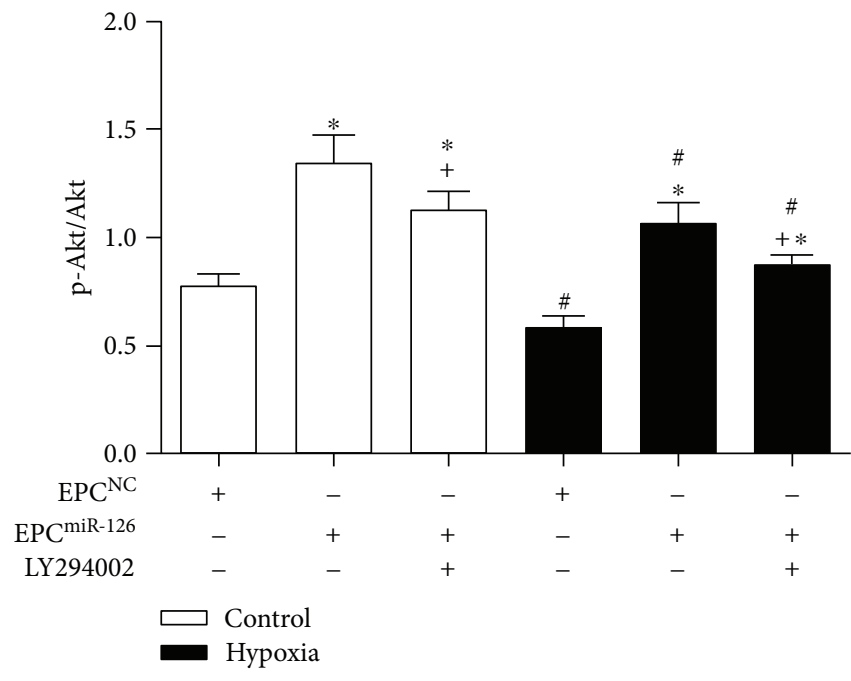

(c)

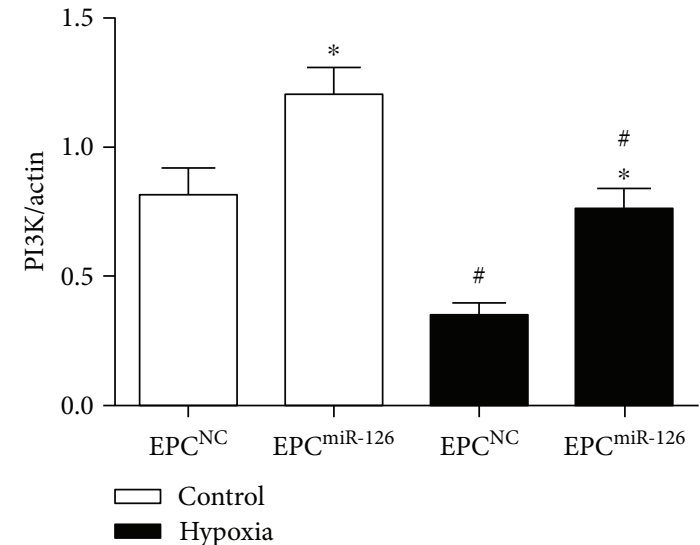

(b)

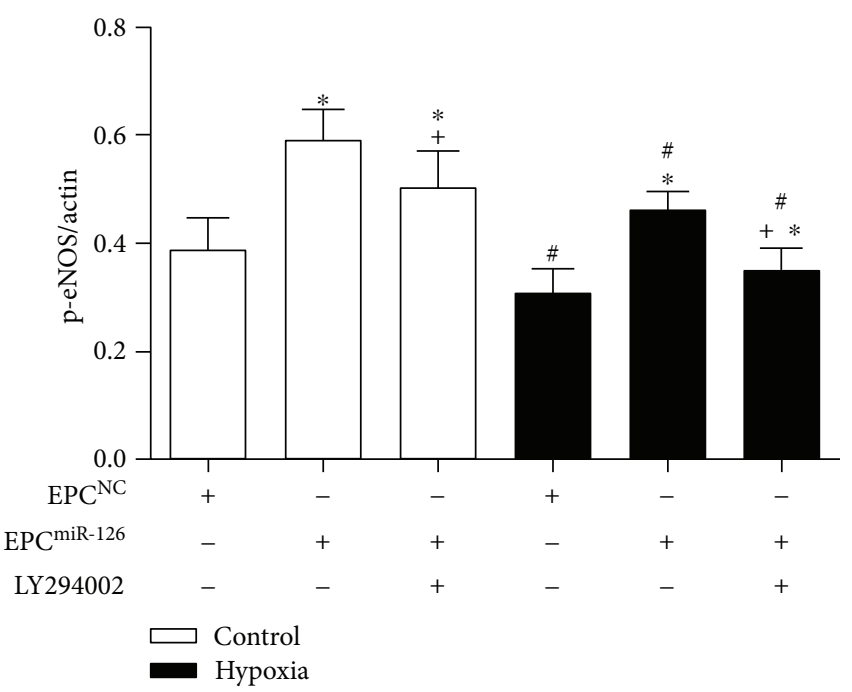

(d)

FIGURE 4: Effects of miR-126 overexpression on the expression of PI3K, p-Akt/Akt, and p-eNOS in EPCs and hypoxia-injured EPCs. (a) Western blot showing expression of PI3K, p-Akt/Akt, and p-eNOS of EPCs. (b-d) Summarized data on the expression of PI3K, p-Akt/ Akt, and p-eNOS. ${ }^{*} p<0.05$ versus $\mathrm{EPC}^{\mathrm{NC}} ;{ }^{+} p<0.05$ versus $\mathrm{EPC}^{\mathrm{miR}-126} ;{ }^{*} p<0.05$ versus control, $n=3 /$ group. 


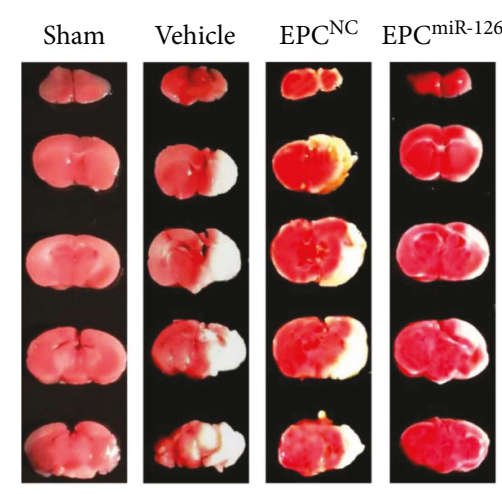

(a)

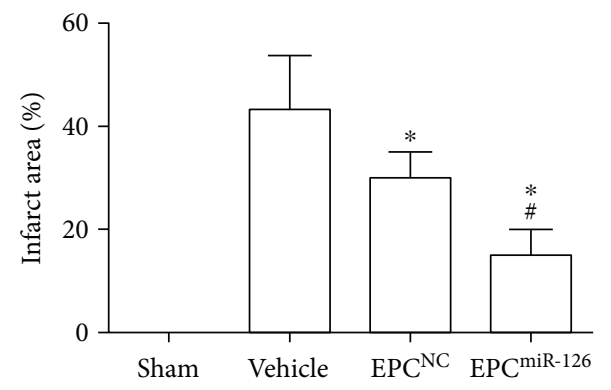

(b)

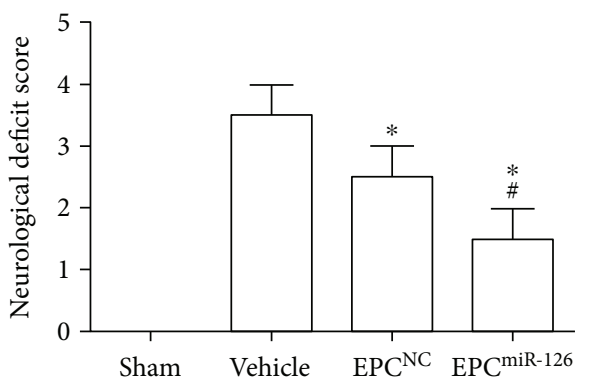

(c)

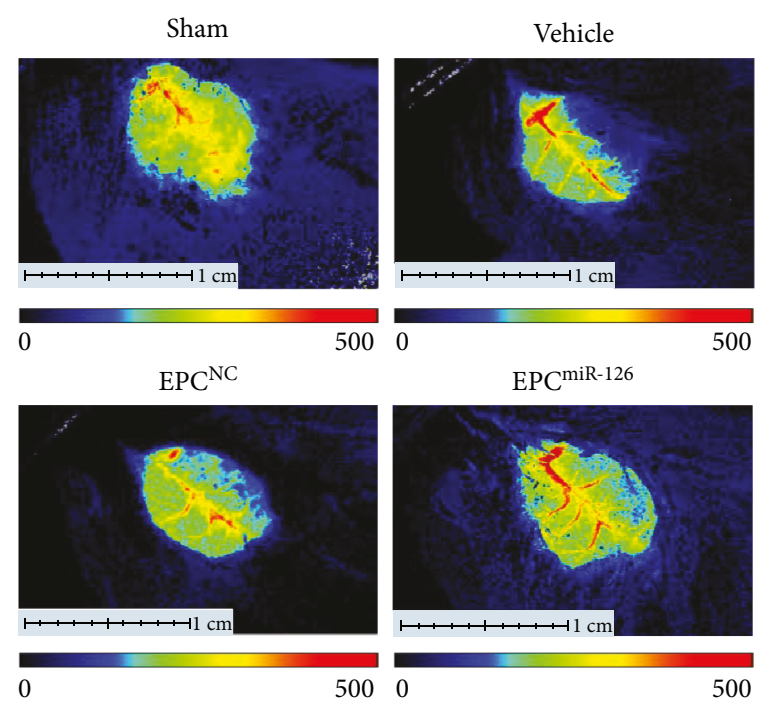

(d)

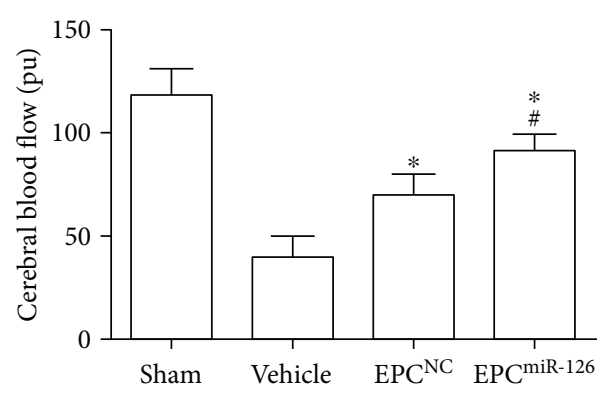

(e)

FIGURE 5: miR-126 priming enhances the efficacy of EPCs in decreasing infarct area and NDS and improving CBF in MCAO mice. (a) Representative images of TTC staining. (b) Quantitative analysis of infarct size in different groups. (c) NDS in different groups. (d) The representative images of CBF. Blue to red represents low to high perfusion. (e) Summarized data on CBF. ${ }^{*} p<0.05$ versus vehicle; ${ }^{\#} p<0.05$ versus $\mathrm{EPC}^{\mathrm{NC}}, n=10$ /group.

infusion of miR-126-primed EPCs was more effective to increase miR-126 expression in cEPCs (versus $\mathrm{EPC}^{\mathrm{NC}}$; $p<0.05$; Figure 7(b)).

\section{Discussion}

Ischemia- and hypoxia-induced energy failure, loss of cellular ion homeostasis, free radical and cytokine-mediated toxicities, inflammation, and disruption of the blood-brain barrier are major pathophysiological processes of ischemic stroke [28]. Current treatments for ischemic stroke mainly rely on vascular recanalization including intravenous or intra-arterial fibrinolysis and interventional treatments [2931]. However, due to the complicated pathophysiological processes, approaches for promoting cerebral recovery following ischemic stroke are limited. Emerging studies demonstrate the effects of EPCs in accelerating cerebral recovery after ischemic stroke [32]. Upon cerebral ischemia, the EPCs are mobilized from the BM to injured blood vessels and ischemic brain tissue. The recruited EPCs then differentiated into mature ECs and secrete protective cellular factors, which participate in endothelialization and replacement of damaged ECs in ischemic tissue [33]. What's more, the EPCs participated in promoting neovascularization by abundant growth factors and cytokines, such as VEGF, SDF, and IGF-1, which can promote EC viability and tube formation, reduce cell apoptosis, and recruit endogenous progenitor cells [34]. Taken together, these findings suggest that EPCs are closely related to the vascular homeostasis and remodeling after ischemic stroke and highlight the potential of EPCs as a cell candidate for regenerative therapy.

However, the in vivo pathological environment may influence the cell fate and therapeutic benefit of EPCs $[7,8,27]$. Reports have showed that the clonogenic and 

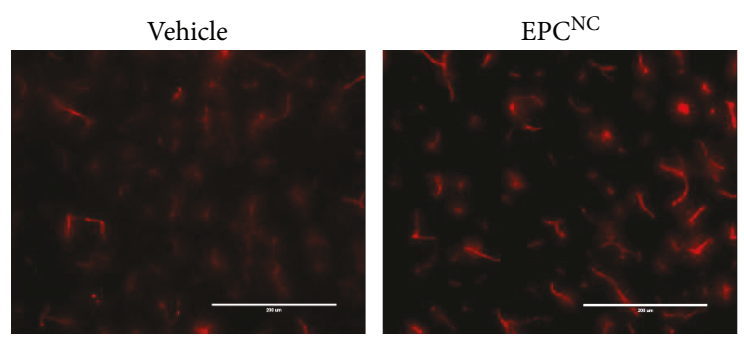

(a)
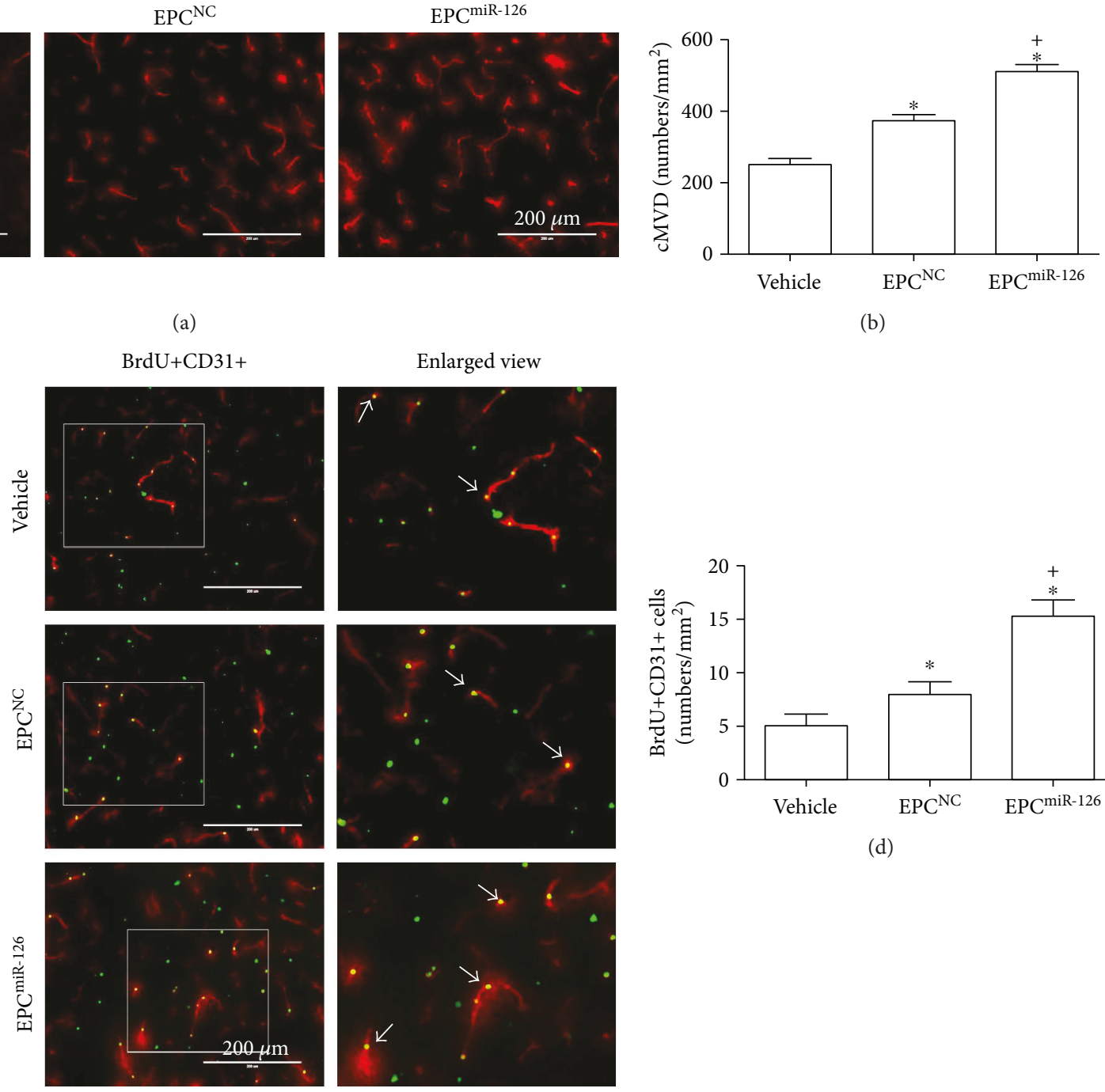

(b)
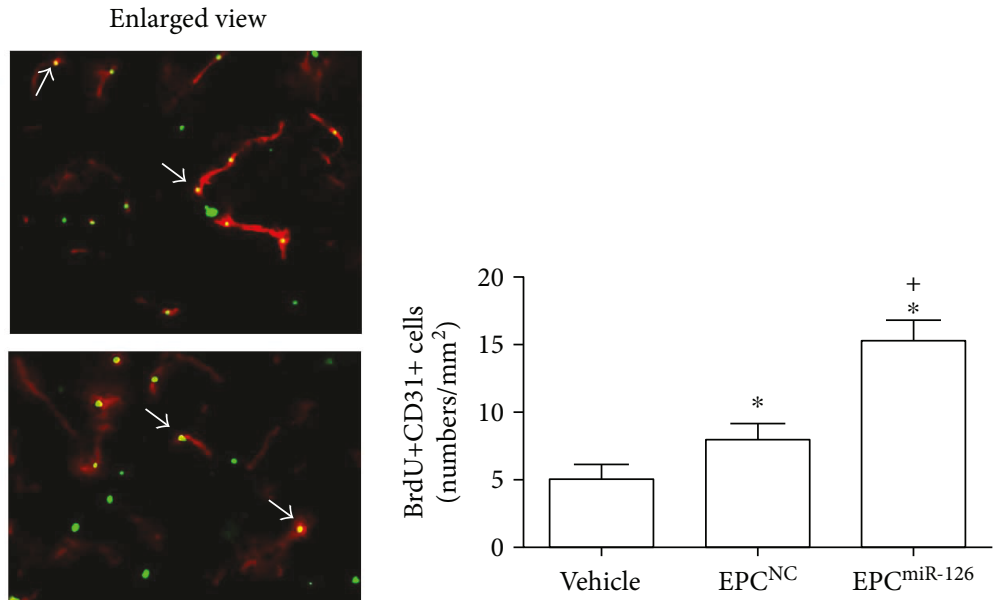

(d)

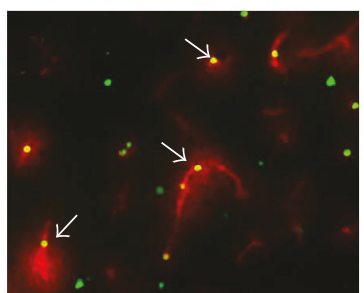

(c)

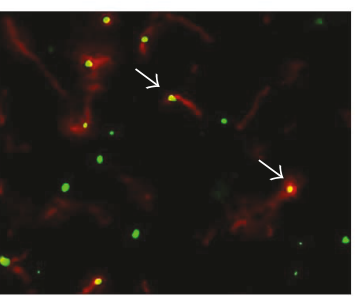

FIGURE 6: miR-126 priming enhances the efficacy of EPCs in increasing cMVD and angiogenesis in the peri-infarct area of MCAO mice. (a) Representative pictures of cMVD (CD31 immunostaining). Scale bar: $200 \mu \mathrm{m}$. (b) Summarized data of cMVD. (c) Representative pictures of angiogenesis (BrdU+CD31+ cells). Red, CD31 for vessel; green, BrdU for new generated cells; pink, double staining. Scale bar, $200 \mu \mathrm{m}$. The enlarged view of double-staining cells is in the left panel of each image. The arrows indicate the double staining cells. (d) Histogram showing the number of BrdU+CD31+ cells. ${ }^{*} p<0.05$ versus vehicle; ${ }^{+} p<0.05$ versus $\mathrm{EPC}^{\mathrm{NC}}, n=10$ /group.

adhesion ability of EPCs was reduced in diabetic patients with peripheral artery disease [35]. Our recent study demonstrated that the migration and tube formation abilities of EPCs were decreased in human renin and angiotensinogen transgenic mice [19]. Another research also demonstrated that the levels of cEPCs were decreased in the plasma of the diabetic mice following ischemic damage [27]. These evidences indicated that the functions of EPCs were closely related to vascular diseases. As a critical factor in cerebral ischemia, hypoxia could impair functions of EPCs [21,36]. Notably, shorter-term hypoxia showed better proliferation, enhancements in tube-like structure formation, and motility of EPCs, as well as mRNA expression of VEGF, CXCR4, PI3K, and Akt [37, 38], while these effects were reversed by prolonged hypoxia [22], which consisted with our present study demonstrated that long-time hypoxia induced EPC dysfunction (decreased proliferation, migration, and tube formation abilities). Thus, in order to enhance the therapeutic effect of EPCs in cerebral ischemia injury, gene transfection and pretreatment have been used to modify EPCs. Various genes such as CXCR4 [39], ACE2 [19], VEGF [40], and eNOS [41] have been transduced into EPCs, which play a beneficial role in improving the therapeutic efficacy of EPCs.

In the present study, we transfected EPCs with miR-126, which is highly enriched in ECs and plays a pivotal role in maintaining vascular integrity and promoting angiogenesis [42]. In vascular injury, the endothelial levels of miR-126 were decreased [43]. The decreased circulating miR-126 has been proved correlated to stroke [44]. Targeted deletion of miR-126 in human umbilical vein vascular endothelial cells upregulates vascular cell adhesion molecule-1, which in turn 

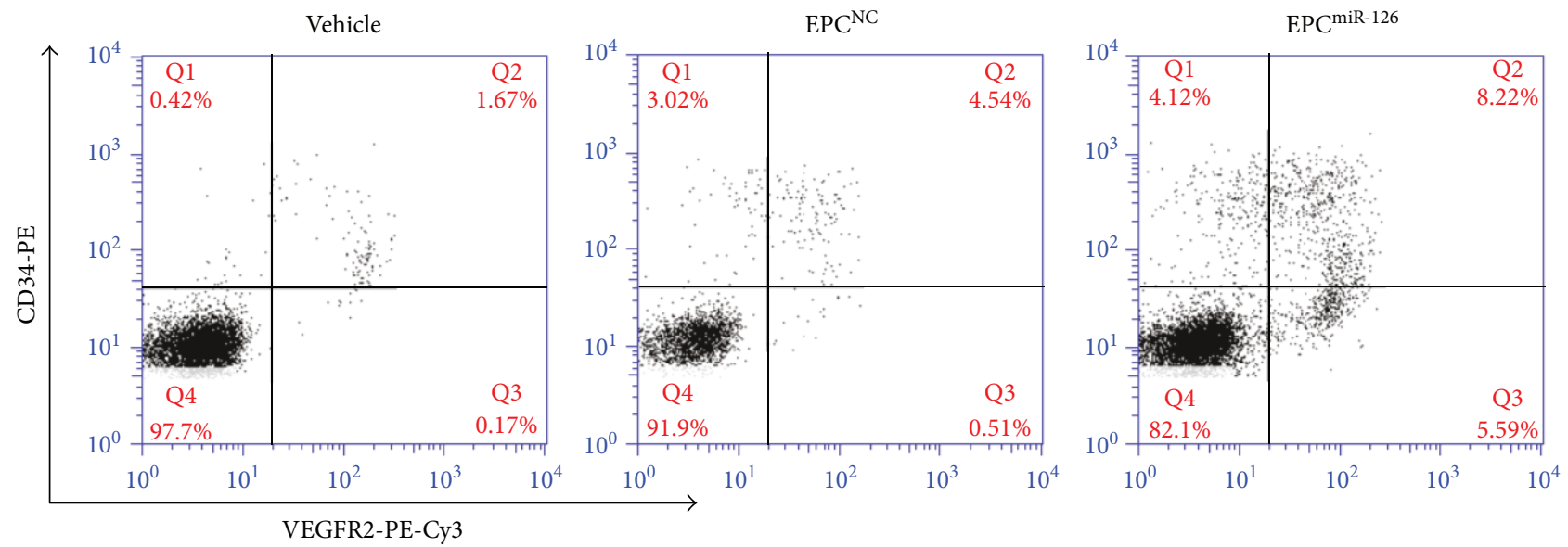

(a)

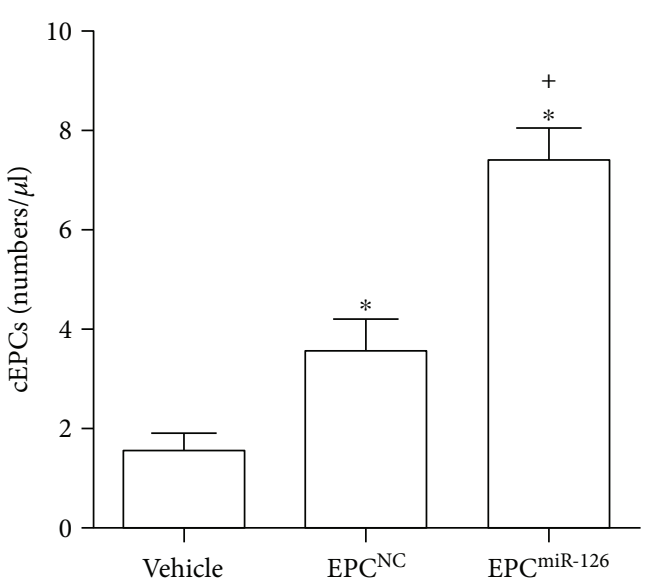

(b)

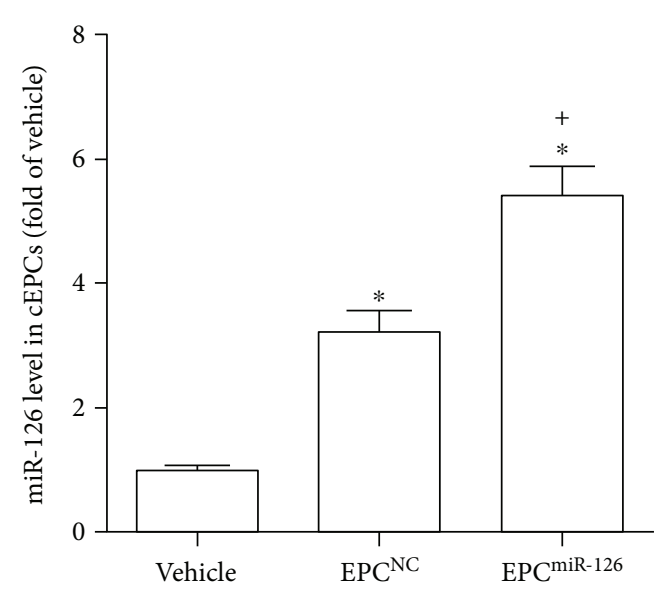

(c)

FIGURE 7: miR-126 priming enhances the efficacy of EPCs in increasing cEPCs and the level of miR-126 of cEPCs in MCAO mice. (a) Representative flow cytometry plot of the levels of cEPCs in each group. (b) Summarized data on the levels of cEPCs. (c) The miR-126 level of cEPCs in MCAO mice in each therapeutic group. ${ }^{*} p<0.05$ versus vehicle; ${ }^{+} p<0.05$ versus $\mathrm{EPC}^{\mathrm{NC}}$. cEPCs: circulating EPCs, $n=10 /$ group.

promotes plaque formation by enhancing leukocyte adherence to the endothelium [45]. The level of miR-126 in EPCs from diabetes mellitus (DM) [18, 46], preeclampsia [47], and coronary artery disease [12] patients was reported to be abnormally lower expressed. This study, for the first time, demonstrated that the level of miR-126 was reduced in EPCs under hypoxic situation, paralleled with inhibited EPC proliferation, migration, and tube formation abilities. The proliferation, migration, and angiogenesis functions of EPCs play an important role in repairing vascular injury by enhancing the ability of EPCs to mobilize from the BM to circulation, migrate into injury or ischemic sites, and promote angiogenesis [48]. Additionally, miR-126 could enhance EPC homing to thrombogenic and ischemic area by targeting PIK3R2 and CXCR4, which plays an important role in the therapeutic effect of EPCs on ischemic diseases $[39,49,50]$. These data suggest that the abnormal expression of miR-126 in the EPCs might be associated with EPC dysfunction and correlated with various vascular diseases. Furthermore, we confirmed that miR-126 overexpression could promote proliferation, migration, and angiogenesis of EPCs in hypoxic or normal condition, which indicated rescuing and enhancing effects of miR-126 on EPC functions.

Hypoxia-induced oxidative stress plays critical role in cerebral ischemic injury, and the mechanisms are relevant to the reduced NO and increased ROS production $[51,52]$. Oxidative stress could impair mobilization and decrease the functionality of EPCs. Evidence suggested that antioxidant treatment prevents the oxidative stress-induced EPC dysfunction [53]. As an important signaling molecule for oxidative stress, ROS plays an important role in endothelial dysfunction via altering vascular integrity [54], while NO could contribute to maintain vascular homeostasis by reducing ROS production [55]. Here, we found that miR126 increased NO production while reduced ROS production in hypoxia-treated EPCs. These indicate that miR-126 decreases oxidative stress of EPCs, which might help improve the effect of EPCs on vascular repair. To further confirm the underlying mechanism of miR-126 involved in regulating ROS generation and NO production of EPCs, we measured the PI3K/Akt/eNOS pathway expression and performed pathway block experiments. Our result showed 
that miR-126 activated PI3K/Akt pathway and upregulated the downstream protein eNOS phosphorylation in EPCs, which was responsible for the production of NO. Furthermore, we found the beneficial effects of miR-126 overexpression on promoting $\mathrm{NO}$ generation and decreasing ROS production in EPCs were partially blocked by PI3K inhibitor. Thus, our findings provide novel evidence that miR-126 could decrease oxidative stress of hypoxia-treated EPCs via activating PI3K/Akt/eNOS signal pathway.

To explore the function of miR-126 overexpression in EPCs in vivo, we investigated the therapeutic efficacy of EPC $^{\text {miR-126 }}$ on MCAO mice model. Our data showed that transfusion of EPC ${ }^{\text {miR-126 }}$ enhanced the beneficial effects of EPCs in attenuating cerebral damage (decreased the infarct volume and NDS) and promoting cerebral repair (increased cMVD, CBF, and angiogenesis). Angiogenesis is a pivot component of wound tissue repair processes. The EPCs are believed to play a pivotal role in angiogenesis, which represents an important endogenous tissue repair mechanism. The EPCs participate in angiogenesis by directly differentiating into matured ECs or by secreting angiogenic factors increasing the viability of resident ECs [56]. In the present study, we stained newly generated ECs with CD31 and BrdU for the index of angiogenesis [39, 57]. Our results showed that the newly generated ECs were increased in the periinfarct area after EPC transfusion, and miR-126 promoted the efficacy of EPCs on angiogenesis. Moreover, new vessel formation after stroke has been shown to replenish blood flow to the ischemic area of the brain [58]. Reestablishing cerebral blood flow in ischemic cerebral microvascular bed contributed to the restoration of neurovascular function and has been set as a potential treatment strategy for ischemic stroke [59]. In this study, we demonstrated for the first time that miR-126 promoted the efficacy of EPCs on ameliorating CBF of ischemia-injured mice, which indicated the beneficial effects of EPC ${ }^{\mathrm{miR}-126}$ on angiogenesis may contribute to the CBF preservation and subsequently neurological functional improvement. Our findings demonstrated that EPC transplantation decreased NDS of ischemic mice, which is consistent with a recent study demonstrating that EPC transplantation improved longterm neurobehavioral outcomes of ischemic stroke [60]. More importantly, we found that miR-126 overexpression could enhance this effect of EPCs. As been proved that angiogenesis contributes to the therapeutic effect of EPCs in promoting neurogenesis after ischemic stroke [32], the beneficial effect of $\mathrm{EPC}^{\mathrm{miR}-126}$ on angiogenesis may also contribute to neuronal functional recovery after ischemic stroke. Taken together, all these data indicate the advantages of miR-126 in enhancing therapeutic efficacy of EPCs for ischemic stroke.

Additionally, we measured the cEPC numbers and the level of miR-126 in cEPCs after transfusion. Accumulating evidence demonstrates that the level of cEPCs is reduced in various stroke risk factors such as hypertension [61], diabetes [62], and atherosclerosis [63]. The number of cEPCs has been recently reported to positively correlate with reendothelialization ability after vascular injury [64]. Thus, we also compared the level of cEPCs between different groups. As a result, we found the level of cEPCs was decreased in MCAO mice. Furthermore, we found that infusion of EPC ${ }^{\text {miR-126 }}$ increased the level of cEPCs and their contained miR-126. As the level of cEPCs negatively correlates with severity of ischemic damage and independently associated with outcome of ischemic stroke [65], the increasing numbers of cEPCs and miR-126 level in cEPCs after transfusion of EPCmiR-126 may be an underlying mechanism involved in the therapeutic efficacy for ischemic stroke.

\section{Conclusion}

Our findings showed that miR-126 rescued/enhanced multiple functions of EPCs, decreased oxidative stress of EPCs via regulating $\mathrm{PI} 3 \mathrm{~K} / \mathrm{Akt} / \mathrm{eNOS}$ pathway, and improved EPC-based therapeutic effects on ischemic stroke. Moreover, circulating EPCs and their carried miR-126 might be important factors closely related to the therapeutic effect of EPCs for ischemic stroke. Our data provide a strong rationale for using miR-126 target to develop vasoprotective drugs and enhance the efficacy of EPC-based therapy.

\section{Conflicts of Interest}

There is no conflict of interests.

\section{Authors' Contributions}

Qunwen Pan, Jieyi Zheng, Donghui Du, Xiaorong Liao, Chunlian Ma, and Yanyu Chen performed the experiments. Wangtao Zhong and Xiaotang Ma designed the experiments. Qunwen Pan and Jieyi Zheng prepared the figures. Qunwen Pan and Donghui Du analyzed the data. Qunwen Pan and Xiaotang Ma drafted the manuscript. Yi Yang, Wangtao Zhong, and Xiaotang Ma edited the manuscript. Qunwen Pan, Jieyi Zheng, and Donghui Du have equal contribution to this manuscript. All authors have read and approved the final version of this manuscript for submission.

\section{Acknowledgments}

This work was supported by the National Natural Science Foundation of China (NSFC, no. 81400360, 81701175), Science and Technology Planning Project of Guangdong Province (no. 2014A020212293), Medical Scientific Research Foundation of Guangdong Province (no. A2016479, A2016485), and Science and Technology Innovation Fund of Guangdong Medical University (no. M2015012, M2016047).

\section{References}

[1] S. Dimmeler and A. M. Zeiher, "Vascular repair by circulating endothelial progenitor cells: the missing link in atherosclerosis?," Journal of Molecular Medicine, vol. 82, no. 10, pp. 671677, 2004.

[2] C. Gandin, C. Widmann, M. Lazdunski, and C. Heurteaux, "MLC901 favors angiogenesis and associated recovery after ischemic stroke in mice," Cerebrovascular Diseases, vol. 42, no. 1-2, pp. 139-154, 2016. 
[3] T. He, L. A. Smith, S. Harrington, K. A. Nath, N. M. Caplice, and Z. S. Katusic, "Transplantation of circulating endothelial progenitor cells restores endothelial function of denuded rabbit carotid arteries," Stroke, vol. 35, no. 10, pp. 2378-2384, 2004.

[4] J. Zhang, W. Zhu, L. Xiao et al., "Lower serum caveolin-1 is associated with cerebral microbleeds in patients with acute ischemic stroke," Oxidative Medicine and Cellular Longevity, vol. 2016, pp. 9026787-9026787, 2016.

[5] H. Zhao, W. Yun, Q. Zhang et al., "Mobilization of circulating endothelial progenitor cells by DL-3-n-butylphthalide in acute ischemic stroke patients," Journal of Stroke and Cerebrovascular Diseases, vol. 25, no. 4, pp. 752-760, 2016.

[6] S. Balaji, A. King, T. M. Crombleholme, and S. G. Keswani, "The role of endothelial progenitor cells in postnatal vasculogenesis: implications for therapeutic neovascularization and wound healing," Advances in Wound Care, vol. 2, no. 6, pp. 283-295, 2013.

[7] P. Jimenez-Quevedo, G. V. Silva, R. Sanz-Ruiz et al., "Diabetic and nondiabetic patients respond differently to transendocardial injection of bone marrow mononuclear cells: findings from prospective clinical trials in "no-option" patients," Revista Española de Cardiología, vol. 61, no. 6, pp. 635-639, 2008.

[8] J. G. Roncalli, J. Tongers, M. A. Renault, and D. W. Losordo, "Endothelial progenitor cells in regenerative medicine and cancer: a decade of research," Trends in Biotechnology, vol. 26, no. 5, pp. 276-283, 2008.

[9] A. E. Pasquinelli, "MicroRNAs and their targets: recognition, regulation and an emerging reciprocal relationship," Nature Reviews Genetics, vol. 13, no. 4, pp. 271-282, 2012.

[10] F. Jansen, X. Yang, M. Hoelscher et al., "Endothelial microparticle-mediated transfer of microRNA-126 promotes vascular endothelial cell repair via SPRED1 and is abrogated in glucose-damaged endothelial microparticles," Circulation, vol. 128, no. 18, pp. 2026-2038, 2013.

[11] H. H. Yang, Y. Chen, C. Y. Gao, Z. T. Cui, and J. M. Yao, "Protective effects of MicroRNA-126 on human cardiac microvascular endothelial cells against hypoxia/reoxygenation-induced injury and inflammatory response by activating PI3K/Akt/ eNOS signaling pathway," Cellular Physiology and Biochemistry, vol. 42, no. 2, pp. 506-518, 2017.

[12] Q. Zhang, I. Kandic, and M. J. Kutryk, "Dysregulation of angiogenesis-related microRNAs in endothelial progenitor cells from patients with coronary artery disease," Biochemical and Biophysical Research Communications, vol. 405, no. 1, pp. 42-46, 2011.

[13] G. Long, F. Wang, H. Li et al., "Circulating miR-30a, miR-126 and let-7b as biomarker for ischemic stroke in humans," $B M C$ Neurology, vol. 13, no. 1, p. 178, 2013.

[14] H. Shi, L. Chen, H. Wang et al., "Synergistic induction of miR126 by hypoxia and HDAC inhibitors in cardiac myocytes," Biochemical and Biophysical Research Communications, vol. 430, no. 2, pp. 827-832, 2013.

[15] C. van Solingen, L. Seghers, R. Bijkerk et al., "Antagomirmediated silencing of endothelial cell specific microRNA-126 impairs ischemia-induced angiogenesis," Journal of Cellular and Molecular Medicine, vol. 13, no. 8a, pp. 1577-1585, 2009.

[16] J. Zhang, Z. Zhang, D. Y. Zhang, J. Zhu, T. Zhang, and C. Wang, "microRNA 126 inhibits the transition of endothelial progenitor cells to mesenchymal cells via the PIK3R2-PI3K/
Akt signalling pathway," PLoS One, vol. 8, no. 12, article e83294, 2013.

[17] T. F. Hansen, C. L. Andersen, B. S. Nielsen et al., "Elevated microRNA-126 is associated with high vascular endothelial growth factor receptor 2 expression levels and high microvessel density in colorectal cancer," Oncology Letters, vol. 2, no. 6, pp. 1101-1106, 2011.

[18] S. Meng, J. T. Cao, B. Zhang, Q. Zhou, C. X. Shen, and C. Q. Wang, "Downregulation of microRNA-126 in endothelial progenitor cells from diabetes patients, impairs their functional properties, via target gene Spred-1," Journal of Molecular and Cellular Cardiology, vol. 53, no. 1, pp. 6472, 2012.

[19] J. Chen, X. Xiao, S. Chen et al., "Angiotensin-converting enzyme 2 priming enhances the function of endothelial progenitor cells and their therapeutic efficacy," Hypertension, vol. 61, no. 3, pp. 681-689, 2013.

[20] Y. Liu, Q. Pan, Y. Zhao et al., "MicroRNA-155 regulates ROS production, NO generation, apoptosis and multiple functions of human brain microvessel endothelial cells under physiological and pathological conditions," Journal of Cellular Biochemistry, vol. 116, no. 12, pp. 2870-2881, 2015.

[21] B. Liu, K. D. Ren, J. J. Peng et al., "Suppression of NADPH oxidase attenuates hypoxia-induced dysfunctions of endothelial progenitor cells," Biochemical and Biophysical Research Communications, vol. 482, no. 4, pp. 1080-1087, 2017.

[22] K. T. Jian, Y. Shi, Y. Zhang, Y. M. Mao, J. S. Liu, and F. L. Xue, "Time course effect of hypoxia on bone marrow-derived endothelial progenitor cells and their effects on left ventricular function after transplanted into acute myocardial ischemia rat," European Review for Medical and Pharmacological Sciences, vol. 19, no. 6, pp. 1043-1054, 2015.

[23] J. W. Yu, Y. P. Deng, X. Han, G. F. Ren, J. Cai, and G. J. Jiang, "Metformin improves the angiogenic functions of endothelial progenitor cells via activating AMPK/eNOS pathway in diabetic mice," Cardiovascular Diabetology, vol. 15, no. 1, p. 88 , 2016.

[24] Q. Pan, X. Liao, H. Liu et al., "MicroRNA-125a-5p alleviates the deleterious effects of ox-LDL on multiple functions of human brain microvessel endothelial cells," American Journal of Physiology Cell Physiology, vol. 312, no. 2, pp. C119-C130, 2017.

[25] Q. Pan, C. He, H. Liu et al., "Microvascular endothelial cellsderived microvesicles imply in ischemic stroke by modulating astrocyte and blood brain barrier function and cerebral blood flow," Molecular Brain, vol. 9, no. 1, p. 63, 2016.

[26] X. Zong, S. Wu, F. Li et al., "Transplantation of VEGFmediated bone marrow mesenchymal stem cells promotes functional improvement in a rat acute cerebral infarction model," Brain Research, vol. 1676, pp. 9-18, 2017.

[27] J. Chen, S. Chen, Y. Chen et al., "Circulating endothelial progenitor cells and cellular membrane microparticles in $d b / d b$ diabetic mouse: possible implications in cerebral ischemic damage," American Journal of Physiology. Endocrinology and Metabolism, vol. 301, no. 1, pp. E62-E71, 2011.

[28] T. M. Woodruff, J. Thundyil, S. C. Tang, C. G. Sobey, S. M. Taylor, and T. V. Arumugam, "Pathophysiology, treatment, and animal and cellular models of human ischemic stroke," Molecular Neurodegeneration, vol. 6, no. 1, p. 11, 2011.

[29] "Effect of intravenous recombinant tissue plasminogen activator on ischemic stroke lesion size measured by computed tomography; The National Institute of Neurological Disorders 
and Stroke (NINDS) rt-PA Stroke Study Group," Stroke, vol. 31, no. 12, pp. 2912-2919, 2000.

[30] C. Castano, L. Dorado, C. Guerrero et al., "Mechanical thrombectomy with the Solitaire AB device in large artery occlusions of the anterior circulation: a pilot study," Stroke, vol. 41, no. 8, pp. 1836-1840, 2010.

[31] M. Lee, K. S. Hong, and J. L. Saver, "Efficacy of intra-arterial fibrinolysis for acute ischemic stroke: meta-analysis of randomized controlled trials," Stroke, vol. 41, no. 5, pp. 932-937, 2010.

[32] A. Taguchi, T. Soma, H. Tanaka et al., "Administration of $\mathrm{CD} 34^{+}$cells after stroke enhances neurogenesis via angiogenesis in a mouse model," The Journal of Clinical Investigation, vol. 114, no. 3, pp. 330-338, 2004.

[33] C. Urbich and S. Dimmeler, "Endothelial progenitor cells: characterization and role in vascular biology," Circulation Research, vol. 95, no. 4, pp. 343-353, 2004.

[34] Y. H. Zhao, B. Yuan, J. Chen et al., "Endothelial progenitor cells: therapeutic perspective for ischemic stroke," CNS Neuroscience \& Therapeutics, vol. 19, no. 2, pp. 67-75, 2013.

[35] U. D. Dincer, "Fetal exposure to a diabetic intrauterine environment resulted in a failure of cord blood endothelial progenitor cell adaptation against chronic hypoxia," Stem Cells and Cloning: Advances and Applications, vol. 8, pp. 1-14, 2015.

[36] K. Zuo, K. Zhi, X. Zhang et al., “A dysregulated microRNA26a/EphA2 axis impairs endothelial progenitor cell function via the p38 MAPK/VEGF pathway," Cellular Physiology and Biochemistry, vol. 35, no. 2, pp. 477-488, 2015.

[37] W. Zhou, W. Zhou, Q. Zeng, and J. Xiong, "MicroRNA-138 inhibits hypoxia-induced proliferation of endothelial progenitor cells via inhibition of HIF- $1 \alpha$-mediated MAPK and AKT signaling," Experimental and Therapeutic Medicine, vol. 13, no. 3, pp. 1017-1024, 2017.

[38] T. Dai, Y. Hu, and H. Zheng, "Hypoxia increases expression of CXC chemokine receptor 4 via activation of PI3K/Akt leading to enhanced migration of endothelial progenitor cells," European Review for Medical and Pharmacological Sciences, vol. 21, no. 8, pp. 1820-1827, 2017.

[39] J. Chen, J. Chen, S. Chen et al., "Transfusion of CXCR4primed endothelial progenitor cells reduces cerebral ischemic damage and promotes repair in db/db diabetic mice," PLoS One, vol. 7, no. 11, article e50105, 2012.

[40] J. X. Yu, X. F. Huang, W. M. Lv et al., "Combination of stromal-derived factor- $1 \alpha$ and vascular endothelial growth factor gene-modified endothelial progenitor cells is more effective for ischemic neovascularization," Journal of Vascular Surgery, vol. 50, no. 3, pp. 608-616, 2009.

[41] S. Kaur, T. R. S. Kumar, A. Uruno, A. Sugawara, K. Jayakumar, and C. C. Kartha, "Genetic engineering with endothelial nitric oxide synthase improves functional properties of endothelial progenitor cells from patients with coronary artery disease: an in vitro study," Basic Research in Cardiology, vol. 104, no. 6, pp. 739-749, 2009.

[42] S. Wang, A. B. Aurora, B. A. Johnson et al., "The endothelialspecific microRNA miR-126 governs vascular integrity and angiogenesis," Developmental Cell, vol. 15, no. 2, pp. 261$271,2008$.

[43] K. Kin, S. Miyagawa, S. Fukushima et al., "Tissue- and plasmaspecific microRNA signatures for atherosclerotic abdominal aortic aneurysm," Journal of the American Heart Association, vol. 1, no. 5, article e000745, 2012.
[44] K. S. Tan, A. Armugam, S. Sepramaniam et al., "Expression profile of MicroRNAs in young stroke patients," PLoS One, vol. 4, no. 11, article e7689, 2009.

[45] T. A. Harris, M. Yamakuchi, M. Ferlito, J. T. Mendell, and C. J. Lowenstein, "MicroRNA-126 regulates endothelial expression of vascular cell adhesion molecule 1," Proceedings of the National Academy of Sciences of the United States of America, vol. 105, no. 5, pp. 1516-1521, 2008.

[46] K. Wu, Y. Yang, Y. Zhong et al., "The effects of microvesicles on endothelial progenitor cells are compromised in type 2 diabetic patients via downregulation of the miR-126/VEGFR2 pathway," American Journal of Physiology Endocrinology and Metabolism, vol. 310, no. 10, pp. E828-E837, 2016.

[47] T. Yan, Y. Liu, K. Cui, B. Hu, F. Wang, and L. Zou, "MicroRNA-126 regulates EPCs function: implications for a role of miR-126 in preeclampsia," Journal of Cellular Biochemistry, vol. 114, no. 9, pp. 2148-2159, 2013.

[48] B. Lapergue, A. Mohammad, and A. Shuaib, "Endothelial progenitor cells and cerebrovascular diseases," Progress in Neurobiology, vol. 83, no. 6, pp. 349-362, 2007.

[49] Q. Meng, W. Wang, X. Yu et al., "Upregulation of MicroRNA126 contributes to endothelial progenitor cell function in deep vein thrombosis via its target PIK3R2," Journal of Cellular Biochemistry, vol. 116, no. 8, pp. 1613-1623, 2015.

[50] B. J. Oh, D. K. Kim, B. J. Kim et al., "Differences in donor CXCR4 expression levels are correlated with functional capacity and therapeutic outcome of angiogenic treatment with endothelial colony forming cells," Biochemical and Biophysical Research Communications, vol. 398, no. 4, pp. 627-633, 2010.

[51] E. H. Lo, M. A. Moskowitz, and T. P. Jacobs, "Exciting, radical, suicidal: how brain cells die after stroke," Stroke, vol. 36, no. 2, pp. 189-192, 2005.

[52] R. X. Yang, J. Lei, B. D. Wang et al., "Pretreatment with sodium phenylbutyrate alleviates cerebral ischemia/reperfusion injury by upregulating DJ-1 protein," Frontiers in Neurology, vol. 8, p. 256, 2017.

[53] E. H. Yao, Y. Yu, and N. Fukuda, "Oxidative stress on progenitor and stem cells in cardiovascular diseases," Current Pharmaceutical Biotechnology, vol. 7, no. 2, pp. 101-108, 2006.

[54] W. J. Zhang, P. X. Li, X. H. Guo, and Q. B. Huang, "Role of moesin, Src, and ROS in advanced glycation end productinduced vascular endothelial dysfunction," Microcirculation, vol. 24, no. 3, 2017.

[55] S. Chuaiphichai, M. J. Crabtree, E. McNeill et al., "A key role for tetrahydrobiopterin-dependent endothelial NOS regulation in resistance arteries: studies in endothelial cell tetrahydrobiopterin-deficient mice," British Journal of Pharmacology, vol. 174, no. 8, pp. 657-671, 2017.

[56] C. Kalka, H. Masuda, T. Takahashi et al., "Transplantation of ex vivo expanded endothelial progenitor cells for therapeutic neovascularization," Proceedings of the National Academy of Sciences of the United States of America, vol. 97, no. 7, pp. 3422-3427, 2000.

[57] H. Kawada, S. Takizawa, T. Takanashi et al., “Administration of hematopoietic cytokines in the subacute phase after cerebral infarction is effective for functional recovery facilitating proliferation of intrinsic neural stem/progenitor cells and transition of bone marrow-derived neuronal cells," Circulation, vol. 113, no. 5, pp. 701-710, 2006.

[58] S. W. Seto, D. Chang, A. Jenkins, A. Bensoussan, and H. Kiat, "Angiogenesis in ischemic stroke and angiogenic effects of 
Chinese herbal medicine," Journal of Clinical Medicine, vol. 5, no. 12, 2016.

[59] J. F. Arenillas, T. Sobrino, J. Castillo, and A. Davalos, “The role of angiogenesis in damage and recovery from ischemic stroke," Current Treatment Options in Cardiovascular Medicine, vol. 9, no. 3, pp. 205-212, 2007.

[60] Y. Fan, F. Shen, T. Frenzel et al., "Endothelial progenitor cell transplantation improves long-term stroke outcome in mice," Annals of Neurology, vol. 67, no. 4, pp. 488-497, 2010.

[61] T. Umemura, J. Soga, T. Hidaka et al., “Aging and hypertension are independent risk factors for reduced number of circulating endothelial progenitor cells," American Journal of Hypertension, vol. 21, no. 11, pp. 1203-1209, 2008.

[62] C. J. M. Loomans, E. J. P. de Koning, F. J. T. Staal et al., "Endothelial progenitor cell dysfunction: a novel concept in the pathogenesis of vascular complications of type 1 diabetes," Diabetes, vol. 53, no. 1, pp. 195-199, 2004.

[63] C. Schmidt-Lucke, L. Rossig, S. Fichtlscherer et al., "Reduced number of circulating endothelial progenitor cells predicts future cardiovascular events: proof of concept for the clinical importance of endogenous vascular repair," Circulation, vol. 111, no. 22, pp. 2981-2987, 2005.

[64] M. Vasa, S. Fichtlscherer, A. Aicher et al., "Number and migratory activity of circulating endothelial progenitor cells inversely correlate with risk factors for coronary artery disease," Circulation Research, vol. 89, no. 1, pp. e1-e7, 2001.

[65] T. Sobrino, O. Hurtado, M. A. Moro et al., "The increase of circulating endothelial progenitor cells after acute ischemic stroke is associated with good outcome," Stroke, vol. 38, no. 10 , pp. $2759-2764,2007$. 


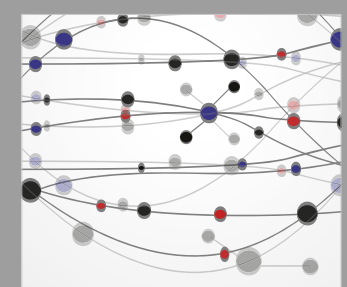

The Scientific World Journal
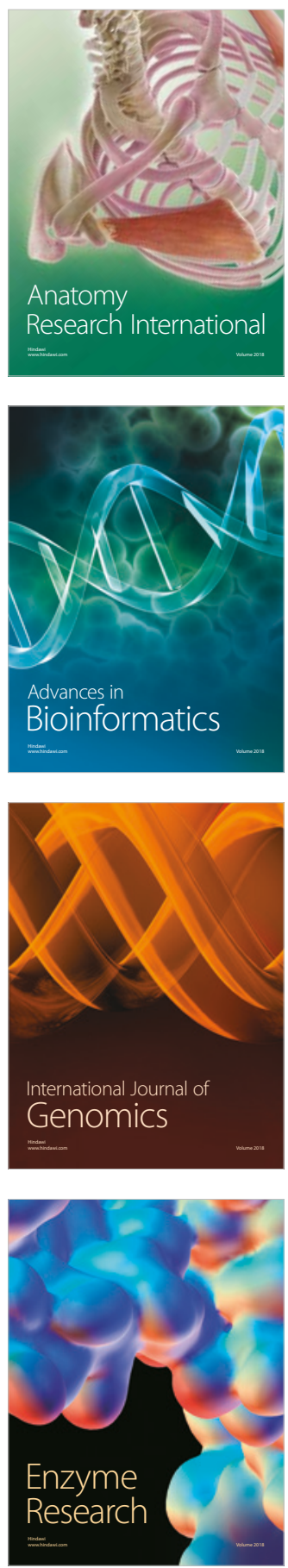
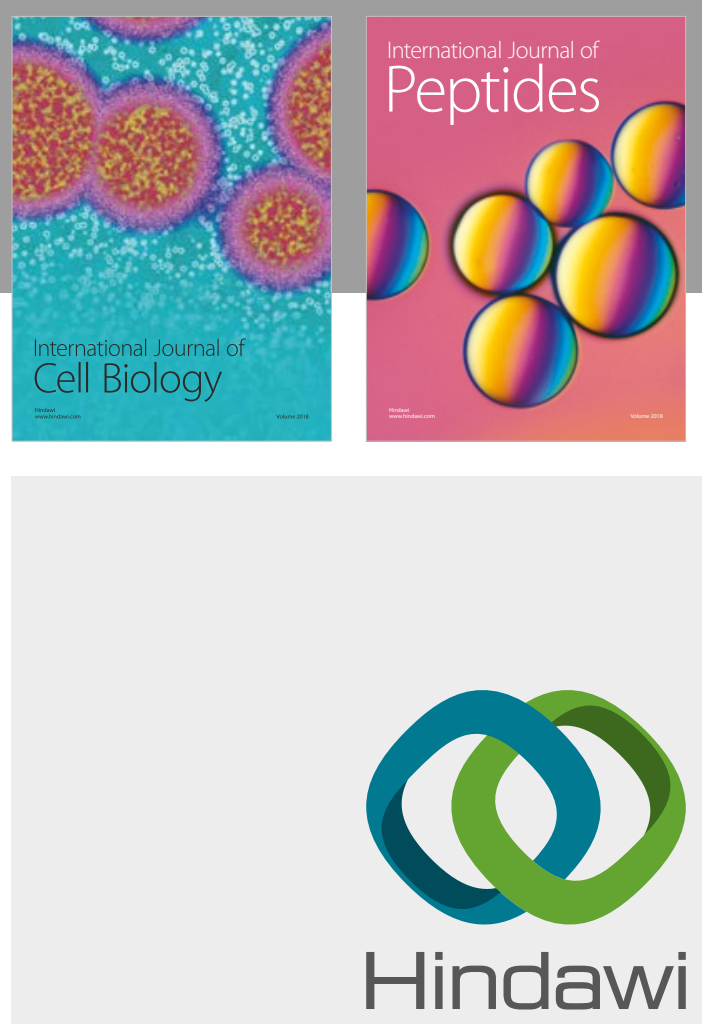

Submit your manuscripts at

www.hindawi.com
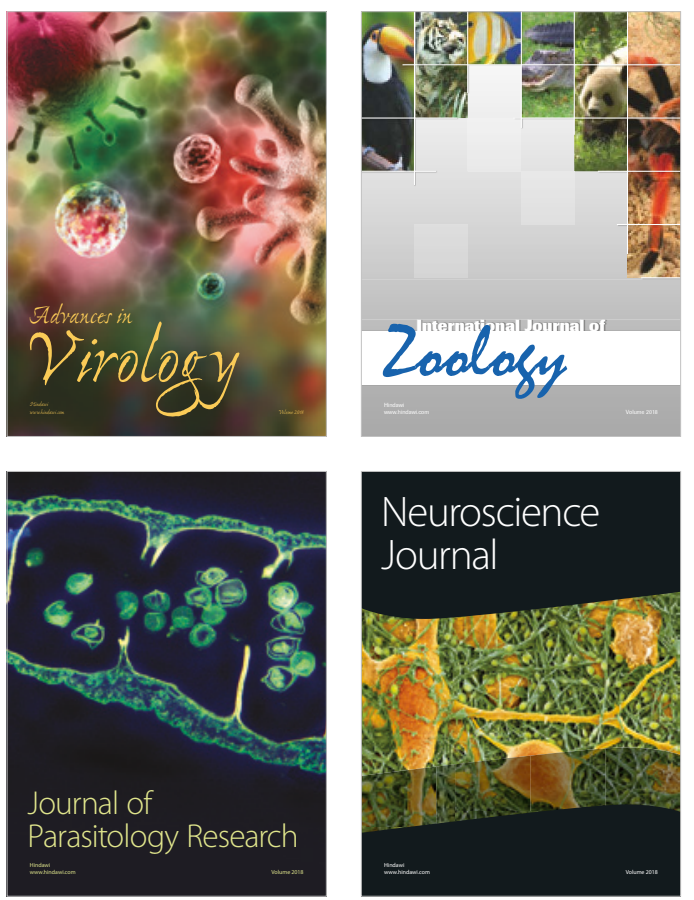
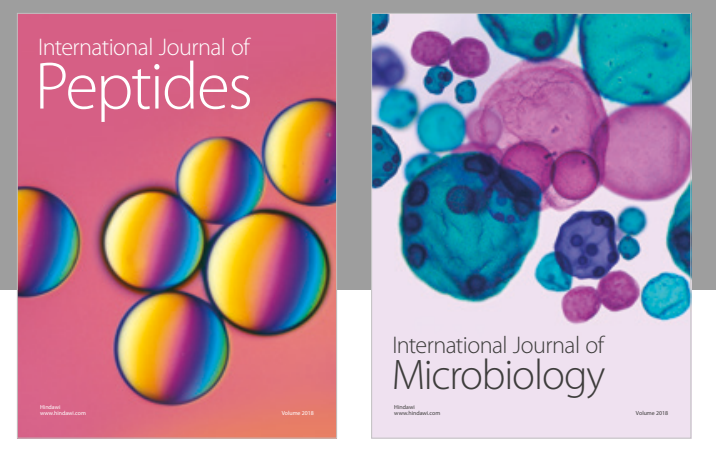

nternational Journal of Microbiology
Journal of
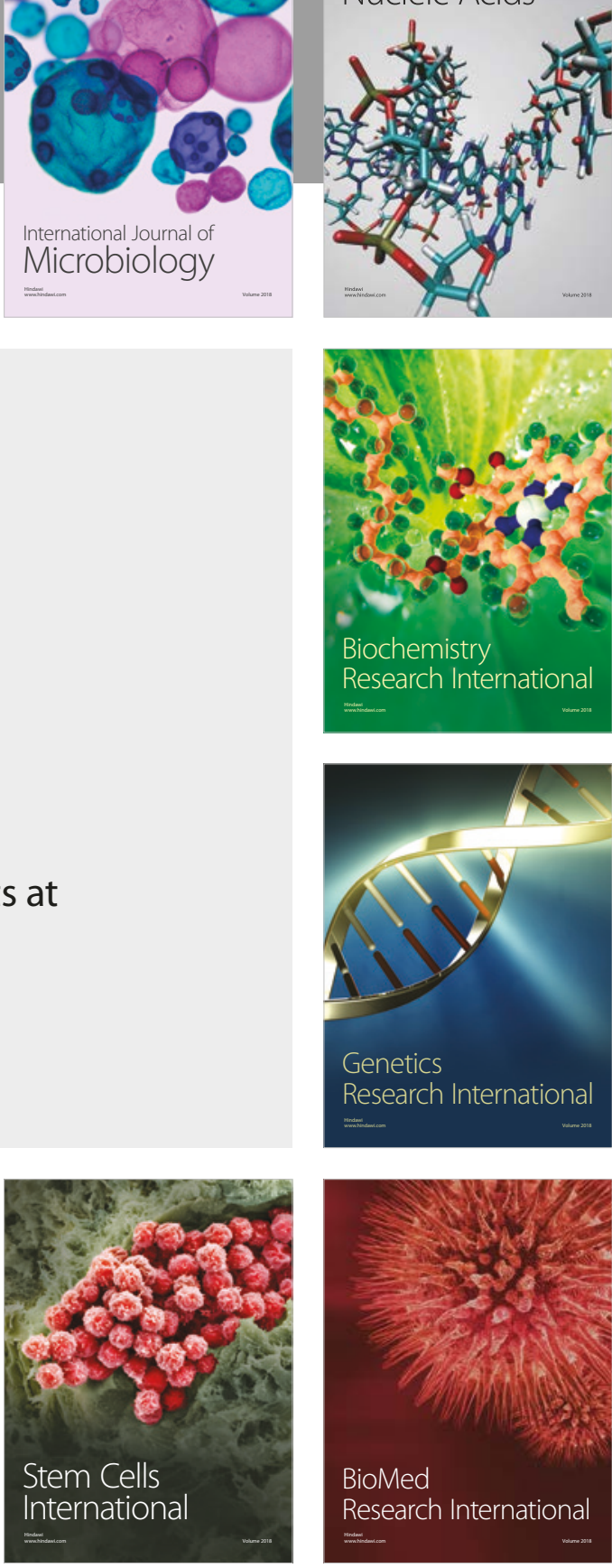
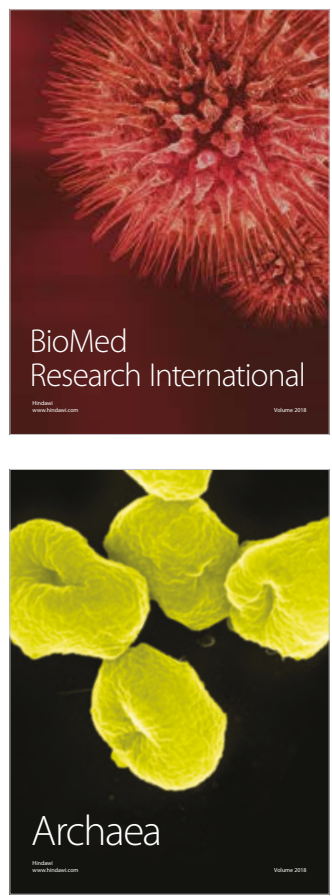